\title{
Analysis Strategy of Powder Diffraction Data with 2-D Detector
}

\author{
Abhik Kumar \\ Office of Science, Science Undergraduate Laboratory Internship (SULI) Program \\ Austin College \\ Stanford Linear Accelerator Center \\ Stanford, California
}

December 16, 2005

Prepared in partial fulfillment of the requirements of the Office of Science, U.S. Department of Energy Science Undergraduate Laboratory Internship (SULI) Program under the direction of Dr. Apurva Mehta and Dr. Samuel Webb in the Synchrotron Radiation Laboratory at the Stanford Linear Accelerator Center.

Participant:

Signature

Research Advisor:

Signature 


\section{Table of Contents}

$\begin{array}{lll}\text { Abstract } & \text { iii }\end{array}$

$\begin{array}{ll}\text { Introduction } & 1\end{array}$

Method $\quad 3$

$\begin{array}{ll}\text { Results } & 11\end{array}$

Discussion and Conclusions 12

$\begin{array}{ll}\text { Acknowledgements } & 13\end{array}$

$\begin{array}{ll}\text { References } & 14\end{array}$

$\begin{array}{ll}\text { Tables and Figures } & 15\end{array}$ 


\begin{abstract}
Analysis Strategy of Powder Diffraction Data with 2-D Detector. ABHIK KUMAR (Austin College, Sherman, TX 75090) SAMUEL WEBB (Stanford Linear Accelerator Center, Stanford, CA 94305) APURVA MEHTA (Stanford Linear Accelerator Center, Stanford, CA 94305).

To gain a clearer understanding of orientation and grain deformation of crystalline materials, x-ray powder diffraction has played an integral role in extracting three-dimensional structural information from one-dimensional diffraction patterns. Powder diffraction models identical geometry to the intersection of a normal right cone with a plane. The purpose of this paper is to develop a general expression defining the conic sections based on the geometry of a powder diffraction experiment. Applying the derived formulation of a diffraction arc to experimental data will give insight to the molecular and structural properties of the sample in question. Instead of using complex three-dimensional Euclidian geometry, we define the problem solving technique with a simpler two-dimensional transformation approach to arrive at the final equation describing the conic sections. Using the diffraction geometry parameters, we can use this equation to calibrate the diffractometer from the diffraction pattern of a known reference material, or to determine the crystalline lattice structure of the compound.
\end{abstract}




\section{INTRODUCTION}

When an X-ray beam falls upon an atom, one of two processes may occur: (1) the beam is absorbed by the atom with the ejection of irradiated electrons, or (2) the beam will scatter [1]. For the purposes of this paper, we will consider the effects of the second case. Experimentally, this process of X-ray scattering, known as diffraction, has been routinely applied towards the understanding of lattice structure. It is capable of quantifying variations in orientation, and grain deformations of a compound [1]. Understanding such phenomena is critical in defining various mechanical properties, such as strength or fatigue resistance, characteristics usually tied to grain microstructure [1]. Once bombarded by the beam, atoms of a crystal channel the electromagnetic waves of the beam onto a 2-D detector plane, capturing a unique and well defined interference curve known as an X-ray diffraction pattern [2] (figure 1).

$\mathrm{X}$-ray powder diffraction has established numerous applications in recent years such as phase identifications, the determination of accurate unit-cell dimensions, and the analysis of structural imperfections. Because of the ability to identify crystal structures from powder data, powder diffraction has served an important role in extracting three-dimensional structural information from one-dimensional patterns to study crystalline materials [2]. Due to a characteristic arrangement of atoms in a crystalline solid, there exists a distinctive $\mathrm{X}$-ray powder diffraction pattern. A normal right cone emerges in powder experiments, centered at the sample. A two-dimensional detector plane intersects the cone at some distance $d$ from the sample. We assign a coordinate grid to the system by which the cone and plane will be defined using traditional Cartesian coordinates (figure 2). We shall refer to the vertical axis on the face of the cone as the $x$ axis, and a horizontal axis, which we will refer to as $y$, bisects this line at a single

point we will call the center. A straight line travels through the apex of the cone through the path 
of the unscattered beam to the face of the cone, precisely where the $x$ and $y$ axes intersect at the center. We will call this third axis the cone’s central axis, and assign it the variable $z$.

Spatially, the orientation of the intersecting plane can assume any position in three dimensions. Depending on the orientation of the plane, a cone may yield one of four curves defined by previous literature: a circle, ellipse, parabola, or hyperbola [4]. We will frequently refer to these curves as 'conic sections'. The purpose of this paper is to develop a completely general expression defining the conic sections based on the geometry of a powder diffraction experiment. Applying the derived formulation of a diffraction arc to actual experimental data will give insight to the molecular and structural properties of the sample in question.

\section{METHOD}

\section{The Method of Transformation}

In lieu of approaching this problem with complex three-dimensional Euclidian geometrics, we define the problem-solving technique in much different terms, using what we will refer to as the method of transformation.

The method of transformation can be simply looked at as the general optical principle of mapping an object's shadow onto a plane. In more analytic terms, we describe a mathematical transform mapping each point on the set of loci of the curve that defines a two-dimensional object from its respective plane, onto an arbitrary plane in space by a dependence relation. This method changes the entire dynamic of the original problem and creates a much easier twodimensional perspective, versus the highly sophisticated (and uneasily visualized) threedimensional approach. 
It is important to recognize the non-linear relation between object and its transformation in certain cases. For example, the transformation of a closed curve will not always resemble a closed transform. This is true simply because when the intersecting plane is rotated a certain magnitude in any direction, the real image on the new plane is distorted in some direction.

Inherent elegance exists in this approach simply because the problem can be broken into two much more transparent two-dimensional problems: a) transformation of the $x$ coordinate, and b) its corresponding $y$. The new points of transformation shall be marked $x^{\prime}$ and $y^{\prime}$ respectively. This method is useful because transformation of a point can occur for any given object of any shape or size. Consider an amorphous object as shown by figure 3 .

By defining an $x$ and $y$ coordinate grid to the object, its complementary point ( $x^{\prime}$ and $\left.y^{\prime}\right)$ can be seen on the new plane of interest. A common analogy of this is seen when the sunlight casts the shadow of any shape onto the planar ground.

\section{Pitch, Tilt, \& Roll}

In any three dimensional system, rotation of a plane can occur about the orthogonal x, y, and $\mathrm{z}$ axes. Similarly, we must devise a way to define the orientation of a plane in space as rotation about these three principle axes. We shall use the terms tilt, pitch, and roll frequently throughout this text to identify rotations of the plane about the $\mathrm{x}, \mathrm{y}$, and $\mathrm{z}$-axes respectively. As an effect of the planar orientation, we will develop a scheme to define the resulting conic sections with simple geometric manipulation.

\section{Pitch}

Pitching $x$ 
First let's consider manipulations of the $x$ case. Figure 3 illustrates the transformation of a locus of points defining any object onto an arbitrary plane. We will call the angle of rotation between them $\beta$. The plane is being rotated about the fixed y axis, which would be coming out of the plane of the paper where $x$ and $x$ ' intersect. Consider the following:

By looking at the problem in two dimensions as figure 4 illustrates, we should solve for $x$ as a function of $x^{\prime}$. We see

$$
\operatorname{Tan} \eta=\frac{x}{d}=\frac{x^{\prime} \operatorname{Cos} \beta}{d+x^{\prime} \operatorname{Sin} \beta}
$$

Therefore,

$$
x=\frac{d x^{\prime} \operatorname{Cos} \beta}{d+x^{\prime} \operatorname{Sin} \beta}
$$

Pitching $y$

Once the $\mathrm{x}$ axis undergoes a pitch at some angle $\beta$, a new change in the $y$ component occurs. Figure 5 shows the cross section of $y$ undergoing a pitch.

As mentioned before, we hope to derive the relationship for $y$ in terms of $\beta$. In doing so, we will have a valid expression for the $y$ component of the curve after the pitch. Because of the common sides $w$ (the length from the source to the object) and $v$ (the length from the object to the plane) shown in both of the previous figures, we can solve for $y$ with respect to $\beta$ (and $x^{\prime}$ ) as shown below.

$$
\frac{y}{w}=\frac{y^{\prime}}{(w+v)}
$$




$$
y=\frac{w}{(w+v)} y^{\prime}
$$

From figure 3 we see again that a common ratio exists.

$$
\begin{gathered}
\frac{x}{w}=\frac{x^{\prime} \operatorname{Cos} \beta}{(w+v)} \\
\frac{w}{(w+v)}=\frac{x}{x^{\prime} \operatorname{Cos} \beta}
\end{gathered}
$$

Substituting this back in for $y$, we get a new expression in terms of $x, x^{\prime}$, and $y^{\prime}$.

$$
y=\frac{x}{x^{\prime} \operatorname{Cos} B} y^{\prime}
$$

Already creating an expression for $x$ as a function of $x^{\prime}$ we substitute equation 1.2, and cancelling out terms, we show

$$
y=\frac{d y^{\prime}}{d+x^{\prime} \operatorname{Sin} \beta} .
$$

\section{Tilt}

Tilting $x$

After defining the object by a pitch, we must now add a tilt in the plane's orientation.

Tilting the plane about the $\mathrm{x}$ axis has an effect on the $x$ and $y$ component of a point on the curve and its transformation map. We shall first consider the $x$ case. In order to derive a valid expression for $x$ undergoing both a pitch and tilt simultaneously, we must look each as a separate process, one occurring after the other. Thus, we can create an equation for a tilted $x$, and substitute it into the initial expression where $x$ has only been pitched (equation 1.2). Let the variable $\gamma$ be the angle of rotation about the $\mathrm{x}$ axis, and we will call the new variable for a tilted $\mathrm{x}$ 
axis $x^{\prime \prime}$. Therefore, the variable $x^{\prime}$ expressed before will be written as a function of $x^{\prime \prime}$. This will give us the expression for $x$ undergoing both pitch and tilt simultaneously as a function with respect to $\beta$ and $\gamma$; as discussed before, once the $x$ component has already experienced a pitch rotation in the $\beta$ direction, $x$ and $x^{\prime}$ are slanted as shown in figure 6 .

Using proportion properties of similar triangles, we see

$$
\frac{x^{\prime}}{w}=\frac{x^{\prime \prime}}{(w+v)}
$$

By algebraic manipulation, we solve for $x$.

$$
x^{\prime}=\frac{w}{(w+v)} x^{\prime \prime}
$$

Based on figure 7, we see that a common relation exists, yet again, with variables $w, v$ during a tilt.

$$
\frac{w}{(w+v)}=\frac{y^{\prime}}{y^{\prime \prime} \operatorname{Cos} \gamma}
$$

Thus, we see a new relation for $x^{\prime}$ in terms of $x^{\prime \prime}, y^{\prime}$, and $y^{\prime \prime}$. Substituting expression 2.3, we can create an expression for $x^{\prime}$ in terms of only $x^{\prime \prime}$ and $y^{\prime \prime}$.

$$
x^{\prime}=\frac{y^{\prime}}{y^{\prime \prime} \operatorname{Cos} \gamma} x^{\prime \prime}
$$

In order to solve a final expression for $x^{\prime}$, we must also solve for $y^{\prime}$ as equation 2.4 dictates. Refer to figure 7. 
Tilting the y axis of the plane allows us to follow the same mathematical procedure as seen earlier for the pitch in $x$. Thus, following the same procedure, our method in solving for the final equation for $y^{\prime}$ (when $y$ has only been pitched) in terms of $y^{\prime \prime}$ (undergoing pitch and tilt) can be described similarly by $x$ in equations 1.1 and 1.2. This results as

$$
y^{\prime}=\frac{d y^{\prime \prime} \operatorname{Cos} \gamma}{d+y^{\prime \prime} \operatorname{Sin} \gamma}
$$

where $\gamma$ is the angle of rotation about the $\mathrm{x}$ axis (the $\mathrm{x}$ axis is pointing out of the plane of the page). By substitution,

$$
x^{\prime \prime}=\frac{d\left(y^{\prime \prime} \operatorname{Cos} \gamma\right)}{d+y^{\prime \prime} \operatorname{Sin} \gamma\left(y^{\prime \prime} \operatorname{Cos} \gamma\right)} x^{\prime \prime}
$$

This gives us

$$
x^{\prime}=\frac{x^{\prime \prime} d}{d+y^{\prime \prime} \sin \gamma} .
$$

With this expression, we will substitute it back into the equation 1.2 to show $x$ after rotation of $x$ and y axes both. We see that

$$
x=\left(\frac{d\left(\frac{d x^{\prime \prime}}{d+y^{\prime} \operatorname{Sin} \gamma}\right) \operatorname{Cos} \beta}{d+\left(\frac{d x^{\prime \prime}}{d+y^{\prime} \operatorname{Sin} \gamma}\right) \operatorname{Sin} \beta}\right)
$$

which simplifies to give us

$$
x=\left(\frac{d x^{\prime \prime} \operatorname{Cos} \beta}{d+y^{\prime \prime} \operatorname{Sin} \gamma+x^{\prime \prime} \operatorname{Sin} \beta}\right)
$$




\section{Tilting $y$}

Refer to figure 8. To arrive at the equation for $y$ after pitch and tilt, we follow the method of transformation further to get the resulting equation for $y$ as a function of $x^{\prime \prime}$ and $y^{\prime \prime}$. To do this the process is simple and follows the same logic as performed with $x$. We must now substitute equations 2.5 and 2.7 into our equation 3.1. Thus, the resulting equation for $y$ should be a function both $x^{\prime \prime}, y^{\prime \prime}, \gamma$, and $\beta$ as desired.

$$
y=\frac{d y^{\prime}}{d+x^{\prime} \operatorname{Sin} \beta}
$$

By substitution,

$$
y=\left(\frac{d\left(\frac{d y^{\prime \prime} \operatorname{Cos} \gamma}{d+y^{\prime \prime} \operatorname{Sin} \gamma}\right)}{d+\left(\frac{d x^{\prime \prime}}{d+y^{\prime \prime} \operatorname{Sin} \gamma}\right) \sin \beta}\right)
$$

Similarly, cancelling out common denominator terms and $d$ coefficients like before, we arrive at

$$
y=\frac{d y^{\prime \prime} \operatorname{Cos} \gamma}{d+y^{\prime \prime} \operatorname{Sin} \gamma+x^{\prime \prime} \operatorname{Sin} \beta}
$$

\section{Roll}

Adding the roll to $x$ and $y$ together

As the final step in arriving at the most general expression for the transformation of points from the object plane to the image plane, we must consider a rotation of the plane about the $\mathrm{z}$ axis, or a roll. This simply is the rotation of the $\mathrm{x}-\mathrm{y}$ coordinate system of the plane some 
angle which we will call $\rho$ (let positive $\rho$ occur in the clockwise direction). The substitution is fairly straight forward. We must redefine $x^{\prime \prime}$ and $y^{\prime \prime}$ identifying the new components as $x^{\prime \prime \prime}$ and $y^{\prime \prime \prime}$. From previous literature we see that the equation for the rotation of the $\mathrm{x}-\mathrm{y}$ coordinate system by the following expression

$$
\begin{aligned}
& x^{\prime \prime}=x^{\prime \prime \prime} \operatorname{Cos} \rho+y^{\prime \prime \prime} \operatorname{Sin} \rho \\
& y^{\prime \prime}=y^{\prime \prime \prime} \operatorname{Cos} \rho-x^{\prime \prime \prime} \operatorname{Sin} \rho
\end{aligned}
$$

where $x^{\prime \prime \prime}$ and $y^{\prime \prime \prime}$ are coordinate axes of the new, rotated plane [2]. Instituting this into the expression is straight forward and involves a simple substitution of the expression of these values into the 2.9 and 3.3 where both tilt and pitch have occurred in $x$ and $y$., incorporating the final rotation of the planar axes in space. From this we see our final expressions for the transformation for the $x$ and $y$ components of any two dimensional object onto a plane which assumes any orientation in three dimensions:

$$
\begin{gathered}
x=\left(\frac{d\left(x^{\prime \prime \prime} \operatorname{Cos} \rho+y^{\prime \prime \prime} \operatorname{Sin} \rho\right) \operatorname{Cos} \beta}{d+\left(y^{\prime \prime \prime} \operatorname{Cos} \rho-x^{\prime \prime \prime} \operatorname{Sin} \rho\right) \operatorname{Sin} \gamma+\left(x^{\prime \prime \prime} \operatorname{Cos} \rho+y^{\prime \prime \prime} \operatorname{Sin} \rho\right) \operatorname{Sin} \beta}\right) \\
y=\left(\frac{d\left(y^{\prime \prime \prime} \operatorname{Cos} \rho-x^{\prime \prime \prime} \operatorname{Sin} \rho\right) \operatorname{Cos} \gamma}{d+\left(y^{\prime \prime \prime} \operatorname{Cos} \rho-x^{\prime \prime \prime} \operatorname{Sin} \rho\right) \operatorname{Sin} \gamma+\left(x^{\prime \prime} \operatorname{Cos} \rho+y^{\prime \prime \prime} \operatorname{Sin} \rho\right) \operatorname{Sin} \beta}\right)
\end{gathered}
$$

\section{Conics}

We have successfully derived a general expression for the transformation map of any given object by taking a complex three dimensional problem and transforming it into two much 
more transparent two dimensional problems. As discussed before, this is analogous to mapping an objects shadow onto a plane. To apply this to the problem of intersecting a cone and a plane, we shall take the case where the object being transformed was a disk. A circular disk can be expressed by the equation $x^{2}+y^{2}=r^{2}$. This is relevant because it models the geometry of X-ray powder diffraction. After substitution, the equation of the conic in terms of the camera length $d$, and the three planar orientation angles is:

$$
\begin{aligned}
& \left(\frac{d\left(x^{\prime \prime \prime} \operatorname{Cos} \rho+y^{\prime \prime \prime} \operatorname{Sin} \rho\right) \operatorname{Cos} \beta}{d+\left(y^{\prime \prime \prime} \operatorname{Cos} \rho-x^{\prime \prime \prime} \operatorname{Sin} \rho\right) \operatorname{Sin} \gamma+\left(x^{\prime \prime \prime} \operatorname{Cos} \rho+y^{\prime \prime \prime} \operatorname{Sin} \rho\right) \operatorname{Sin} \beta}\right)^{2}+ \\
& \left(\frac{d\left(y^{\prime \prime \prime} \operatorname{Cos} \rho-x^{\prime \prime \prime} \operatorname{Sin} \rho\right) \operatorname{Cos} \gamma}{d+\left(y^{\prime \prime \prime} \operatorname{Cos} \rho-x^{\prime \prime \prime} \operatorname{Sin} \rho\right) \operatorname{Sin} \gamma+\left(x^{\prime \prime \prime} \operatorname{Cos} \rho+y^{\prime \prime \prime} \operatorname{Sin} \rho\right) \operatorname{Sin} \beta}\right)^{2}=r^{2}
\end{aligned}
$$

Referring back to figure 4, we can now define the radius of the base of the cone, $r$. We see the radius is a length $x(x=r)$, which for the purposes of clarity, we can redefine as a function using the variable $\alpha$, which is typically used to describe the half angle of the cone. Before, we defined the half-angle with some variable angle $\eta$. Thus, it when $x=r, \eta=\alpha$. Because $\operatorname{Tan} \alpha=r / d, r=d \operatorname{Tan} \alpha$. Our final resulting equation in the most general of terms is:

$$
\begin{gathered}
\left(\frac{d\left(x^{\prime \prime \prime} \operatorname{Cos} \rho+y^{\prime \prime \prime} \operatorname{Sin} \rho\right) \operatorname{Cos} \beta}{d+\left(y^{\prime \prime \prime} \operatorname{Cos} \rho-x^{\prime \prime \prime} \operatorname{Sin} \rho\right) \operatorname{Sin} \gamma+\left(x^{\prime \prime \prime} \operatorname{Cos} \rho+y^{\prime \prime \prime} \operatorname{Sin} \rho\right) \operatorname{Sin} \beta}\right)^{2}+ \\
\left(\frac{d\left(y^{\prime \prime \prime} \operatorname{Cos} \rho-x^{\prime \prime \prime} \operatorname{Sin} \rho\right) \operatorname{Cos} \gamma}{d+\left(y^{\prime \prime \prime} \operatorname{Cos} \rho-x^{\prime \prime \prime} \operatorname{Sin} \rho\right) \operatorname{Sin} \gamma+\left(x^{\prime \prime \prime} \operatorname{Cos} \rho+y^{\prime \prime \prime} \operatorname{Sin} \rho\right) \operatorname{Sin} \beta}\right)^{2}=(d \operatorname{Tan} \alpha)^{2}
\end{gathered}
$$


The next step is to test the equation's consistency and accuracy. For the purposes of this description, we will only consider two variables; the orientation of the plane some angle $\beta$, and the camera length $d$ (a constant). Because of the lateral symmetry of the cone (and for simplicity), we will assign $\gamma$ to equal 0 , and we will also assume no rotation of the coordinate system in which case $\rho$ is equal to 0 . In doing so, we will explore if the equation does indeed represent the conic sections.

As described before, conic sections take on four forms: a circle, ellipse, parabola, and hyperbola. When a plane intersects a cone, these curves form on the face of the cone as a result depending on the planes angle of intersection relative to the cone. When there is no angle of intersection ( $\beta=0$ ), a circle results. This is one of two special cases that we will call critical points. The parabola is the other special case and can only occur under a given condition; the angle of the intersecting plane is complementary to the half angle of the cone, that is, $\beta=90$ $\alpha$. When this is true, the plane is parallel to the nappe of the cone. It is considered a critical point because it is the first angle where the resulting conic section changes from a closed curve to and open curve. In between these two critical points, $(0<\beta<90-\alpha)$ the curve developed is an ellipse. As a result, if we substitute this angle into the equation, it should create the curve of an ellipse. Also, when the angle of intersection is greater than $90-\alpha$, the resulting conic should be a hyperbolic curve (Table 1).

\section{RESULTS}

Table 1 displays the angular limits and the resulting conic sections. Figure 9a shows the result of the equation in graphical form given defined geometric parameters. The half-angle for scattering $\alpha=30$, and the values for $\rho$ and $\gamma=0$. The variable angle of intersection was $\beta$ 
which is shown ranging from 0 to 80 degrees in increments of 10 degrees. As the figure shows, the resulting conic is a circle for all angular values of 0 , and a parabola when $\beta=60$ (or 90- $\alpha$ ). In between these limits, the plot shows variations of ellipses. Above $\beta=60$, a hyperbola occurs. Figure $9 \mathrm{~b}$ shows the resulting curves when a tilt component is added to the plane in the form of angle $\gamma$. The values for $\alpha=30$ degrees and $\beta=40$ degrees were constant. The variable parameter was $\gamma$, ranging from 0 to 80 degrees in increments of 10 . The plot shows the resulting conic section, but with a new orientation as opposed to figure 9a. The final plot (figure 9c) illustrates the implementation of $\rho$. With constant values for $\alpha, \beta$, and $\gamma$, we see that a change in $\rho$ simply rotates the curve about the original origin, as the figure shows.

\section{DISCUSSION AND CONCLUSION}

Using a nontraditional method, the results suggest an accurate and consistent equation satisfying our purpose. Based on the resulting figures referred to in the results, we see that the equation defines general expression for the four conic sections, as a result of the intersection of a cone and a plane which is relevant to understanding diffraction patterns for powder data. This shows that the method of transformation was in fact an effect strategy in tackling the problem, and we were able to limit the expression to defined system parameters.

While approaching this problem geometrically, it is fundamental to notice a few trends. As the angles increase (from 0:90- $\alpha$ ) the semi-major axis defining the length of the ellipse (and the location if its foci) becomes larger. From this we can deduce how the conic sections are actually formed geometrically. Because the foci of a circle are the same point, the center is the foci. As the angle of pitch or tilt increases, the foci move apart, essentially skewing the center of the closed curve. This was evident when mapping the shadow of the disk onto the plane as 
shown in the plots. The center finally moves enough to open the curve and make the center go to infinity as seen with the open curves of the parabola and hyperbolic curves. We see that when substituting in values that satisfy a respective conic section, the general equation should reduce to the form of a general equation of such a curve in two dimensions. For example, when substituting in 0 for all angular values, the plotted curve should be a circle. The equation reduces to the form of $x^{2}+y^{2}=r^{2}$, which is clearly the equation of the circle. This is consistent for all the conic sections.

As shown in the plots, the variations of the tilt and pitch define which of a circle, ellipse, parabola, hyperbola appear. It is important to note that symmetry of the problem. Both angles $\beta$ and $\gamma$ are interchangeable.

The logic applied suggested an unconventional approach at the problem. However, with such an equation, the analysis of microdiffraction data becomes much more easily understood. We now hope to fit existing diffraction pattern data to the equation. Knowing the diffraction geometry parameters, we can calculate the scatter angle value for $\alpha$ which is vital to understand the lattice structure of the sample and its grain structure. The equation can also be applied in the reverse. Based on the shape of the conic Sections from a diffraction pattern of a known reference material, diffraction geometry parameters can be desired, and therefore the diffractometer calibrated. 


\section{ACKNOWLEDGEMENTS}

I would like to thank the U.S. Department of Energy and the Stanford Linear Accelerator Center for the opportunity to engage in such dynamic work as part of a SULI program that is dedicated to developing tomorrow's leaders in science. Special thanks are extended to Dr. Apurva Mehta, an enthusiastic mentor, whose willingness to work with me and encouragement for independent critical thinking proved to challenge me thoroughly and help me learn, grow, and mature as an emerging student researcher. I would also like to thank Dr. Samuel Webb for all his continued help, enthusiasm and support.

\section{REFERENCES}

[1] B.E. Warren, $\underline{X-R a y ~ D i f f r a c t i o n, ~ N e w ~ Y o r k: ~ D o v e r ~ P u b l i c a t i o n s, ~ I n c ., ~} 1969$.

[2] Alan C. Tribble, Princeton Guide to Advanced Physics, Princeton New Jersey: Princeton University Press, 1996.

[3] Jay M. Pasachoff, Physics, Boston: Little, Brown and Company, 1987.

[4] J.W. Downs, Practical Conic Sections, Santa Clara, California: Dale Seymour Publications, 1998. 


\section{Tables}

\begin{tabular}{|c|c|}
\hline ANGLE: $\quad \beta$ or $\gamma$ & Conic Section \\
\hline $0($ both $)$ & Circle \\
\hline$>0$ and $<(90-\alpha)$ & Ellipse \\
\hline $90-\alpha$ & Parabola \\
\hline$>(90-\alpha)$ & Hyperbola \\
\hline
\end{tabular}

Table 1. Conditions for conic sections with angular limits.

\section{Figures}

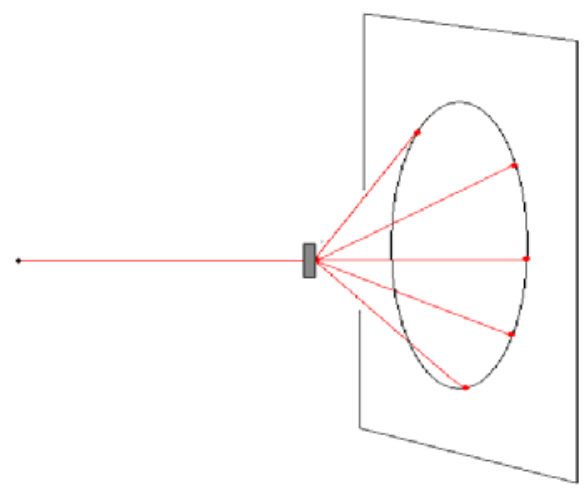

Figure 1. X-Ray diffraction scattering from 3-D sample to 2-D detector

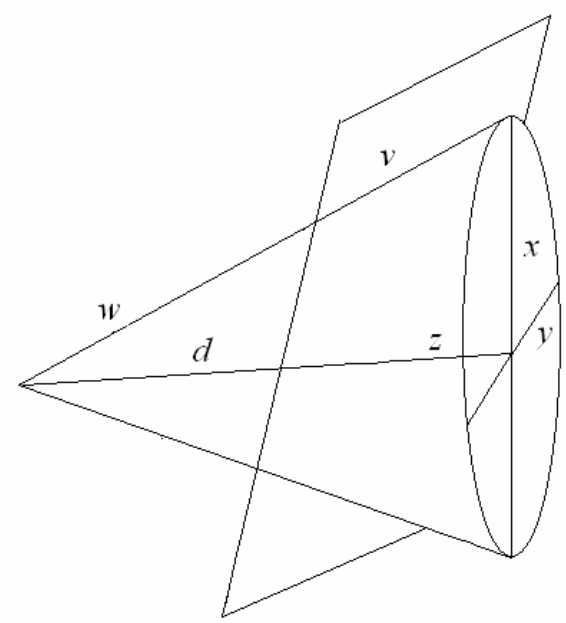

Figure 2. Intersection of a cone and a plane. 


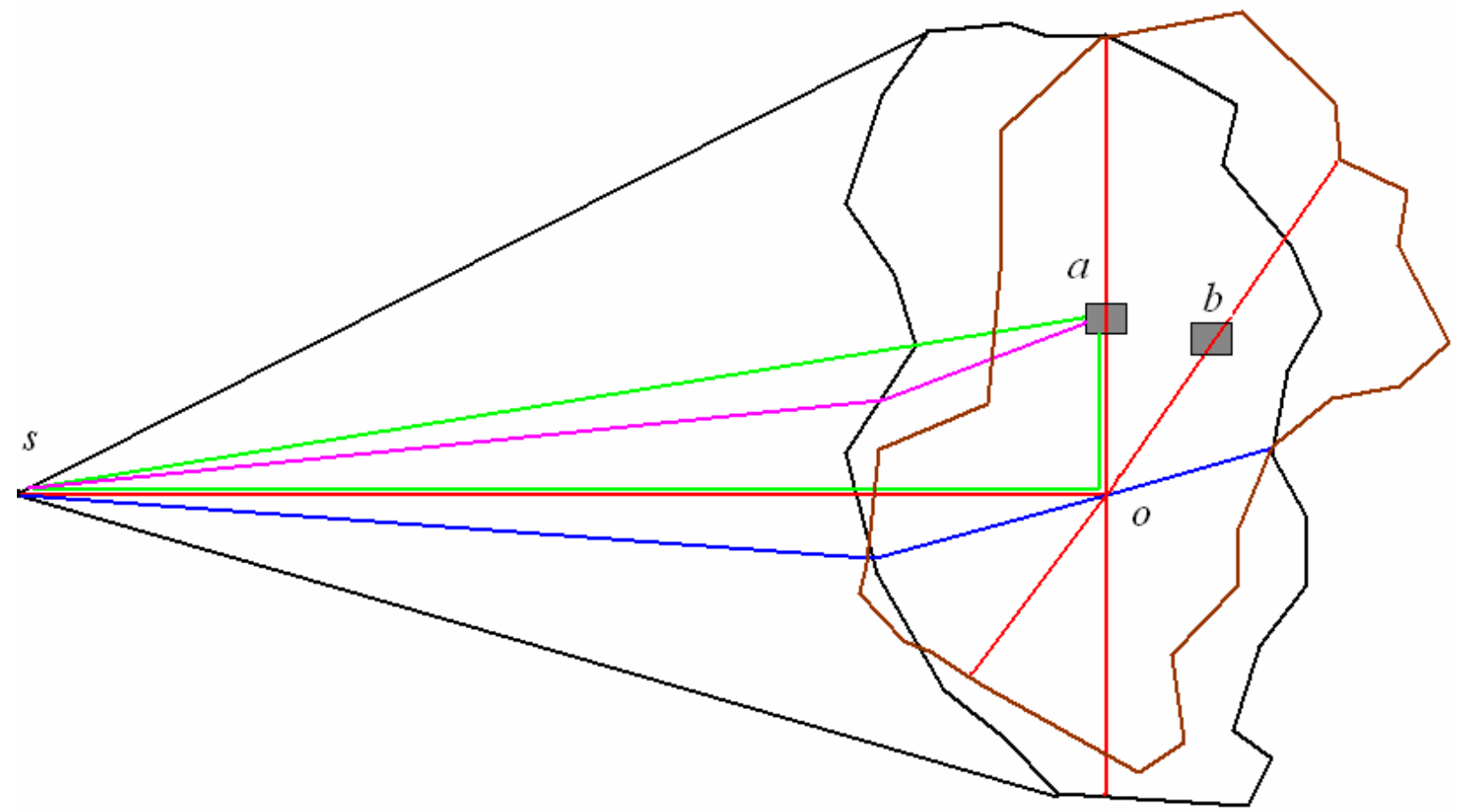

Figure 3. Transformation of a point on any object (point $b$ is the resulting transform of point $a$ ). The brown curve represents an arbitrary plane. 


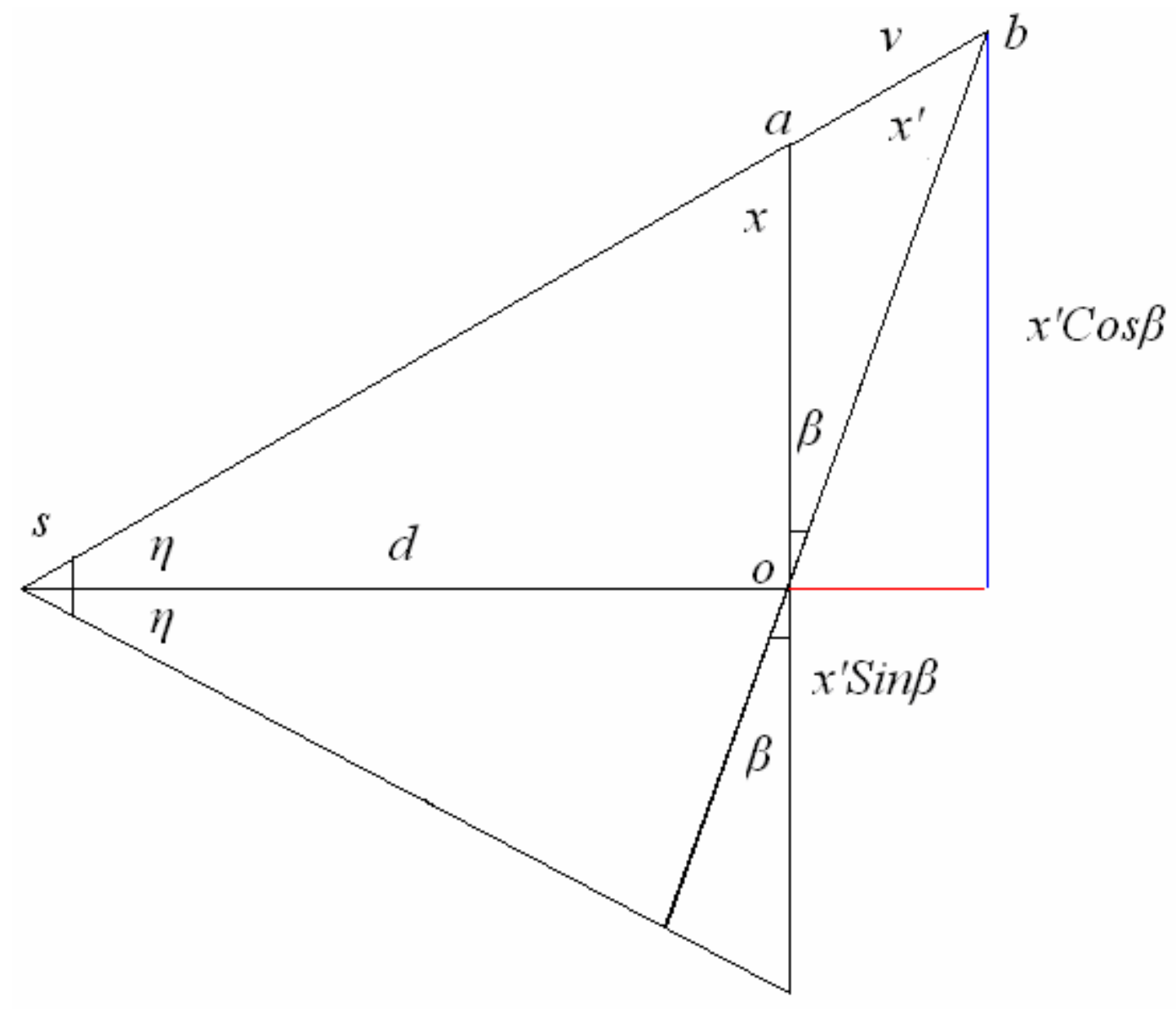

Figure 4. A cross section of the pitch transformation of $x$ onto $x^{\prime}$. Note the red and blue lines are extrapolations of the figure geometry. Let $\eta$ be the scatter angle. The camera length distance from the sample to the plane is denoted by the variable $\mathrm{d}$. 


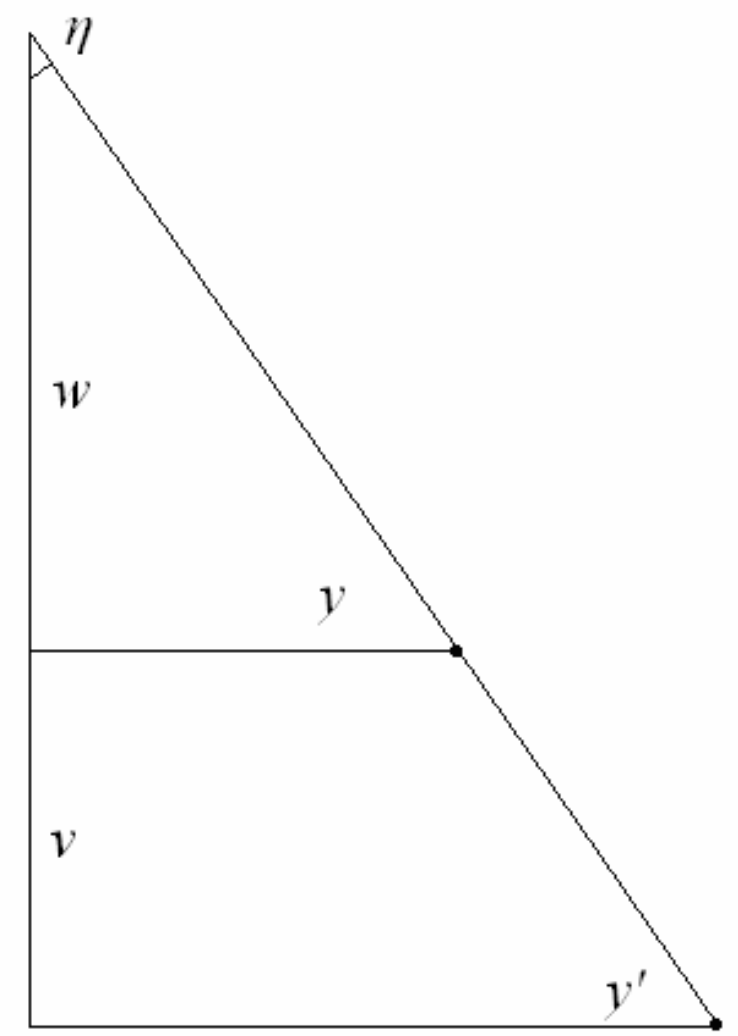

Figure 5. The cross-section of $y$ after pitched.

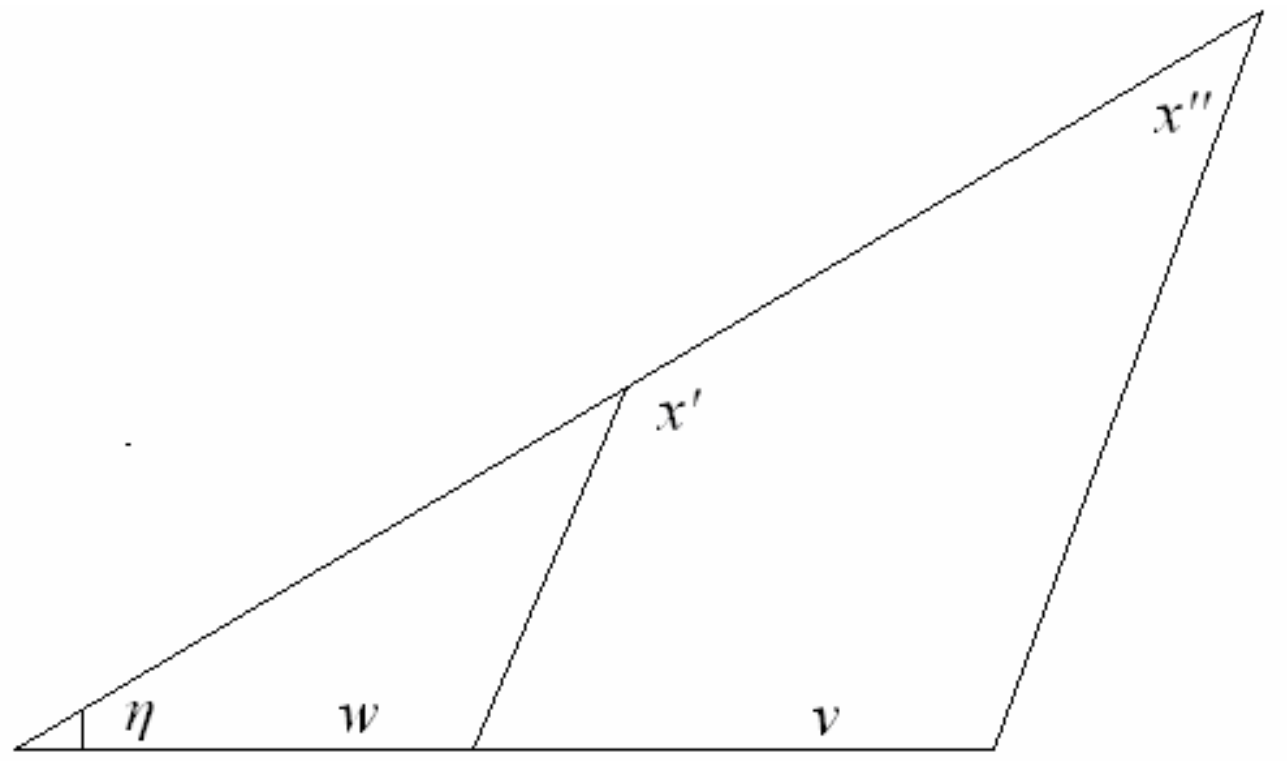

Figure 6. Cross-section of $x$ after pitch and tilt. The triangles are similar. 


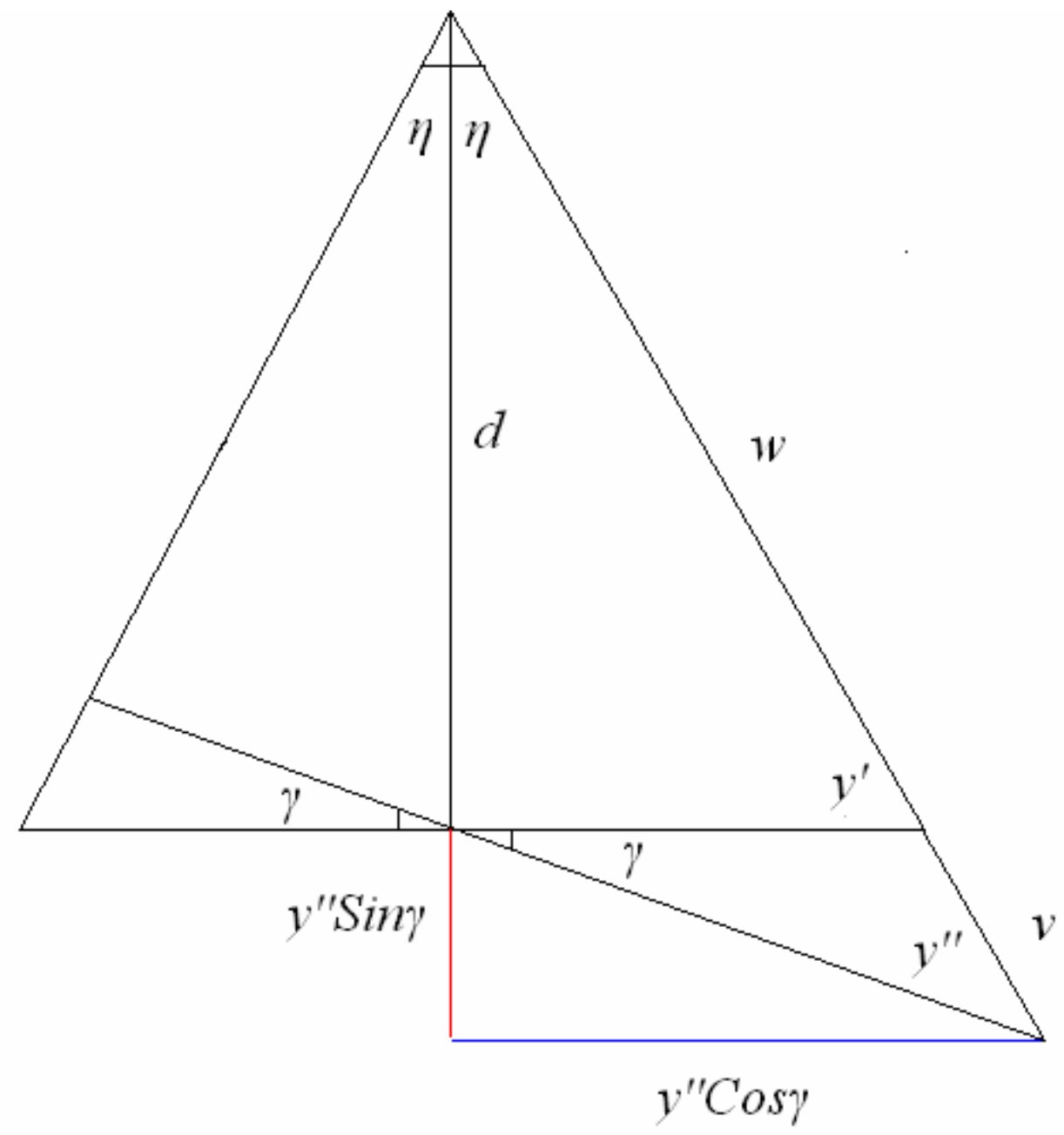

Figure 7. Cross-section of the tilt transformation of $y$ onto $y^{\prime}$. Note the red and blue lines are extrapolations of the figure geometry. 


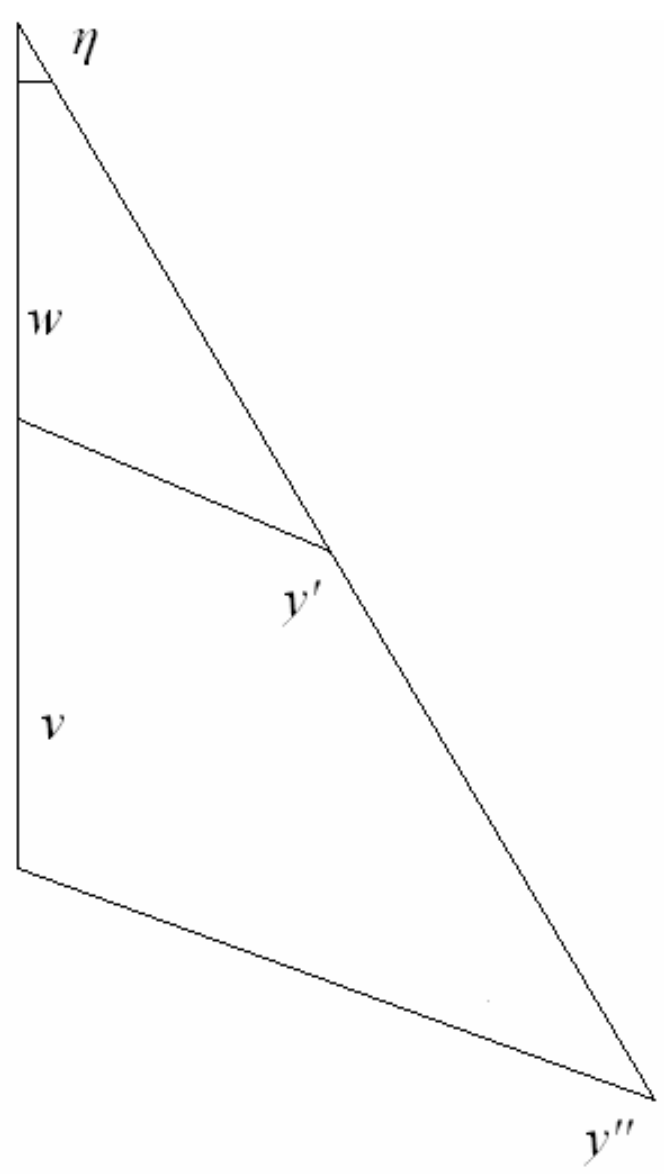

Figure 8. Cross-section of $y$ after tilt and pitch.

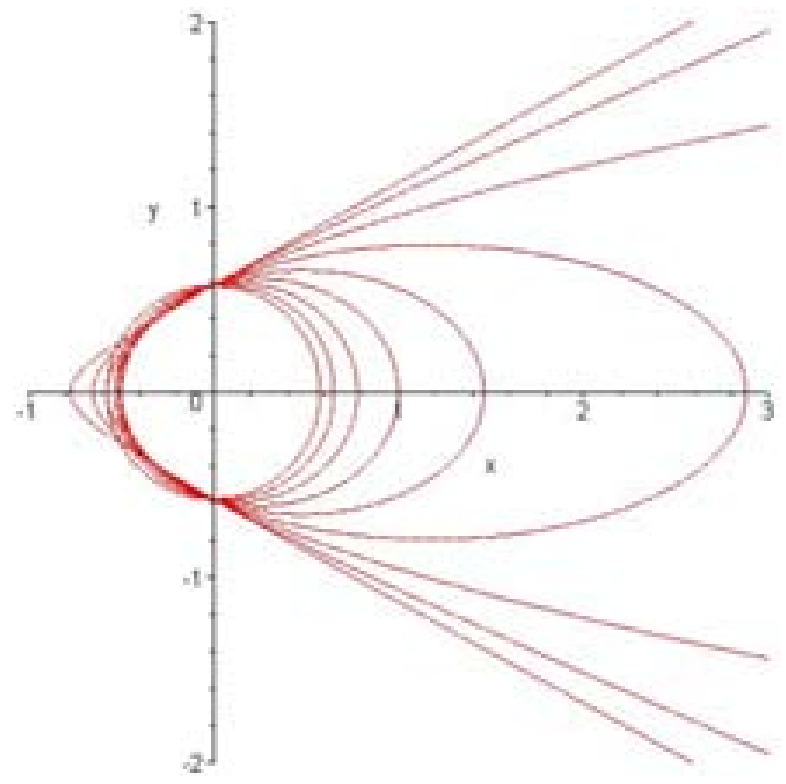

Figure 9a. Plot of resulting conic sections. $\alpha=30, \beta=40, \gamma=0, \rho=0$. 


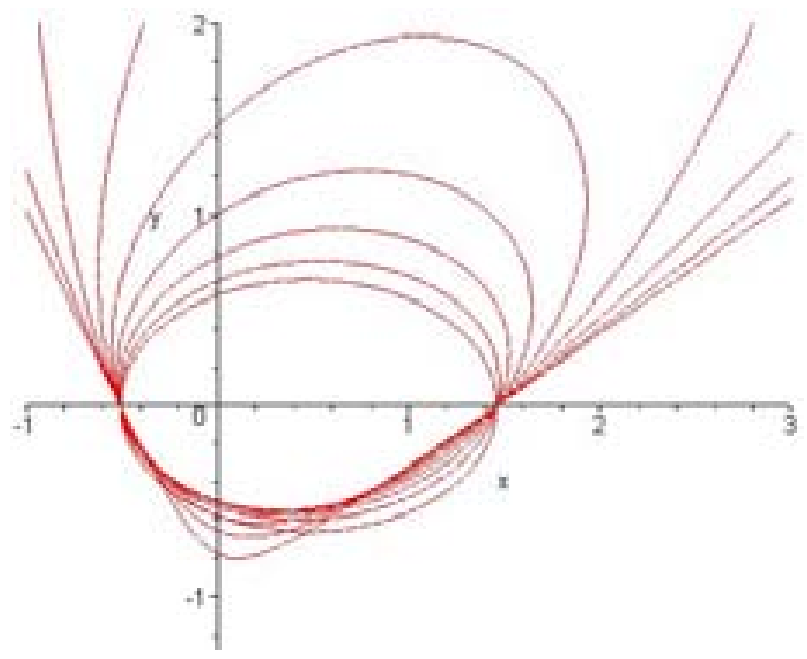

Figure 9b. Plot of resulting conic with the addition of $\gamma=40$.

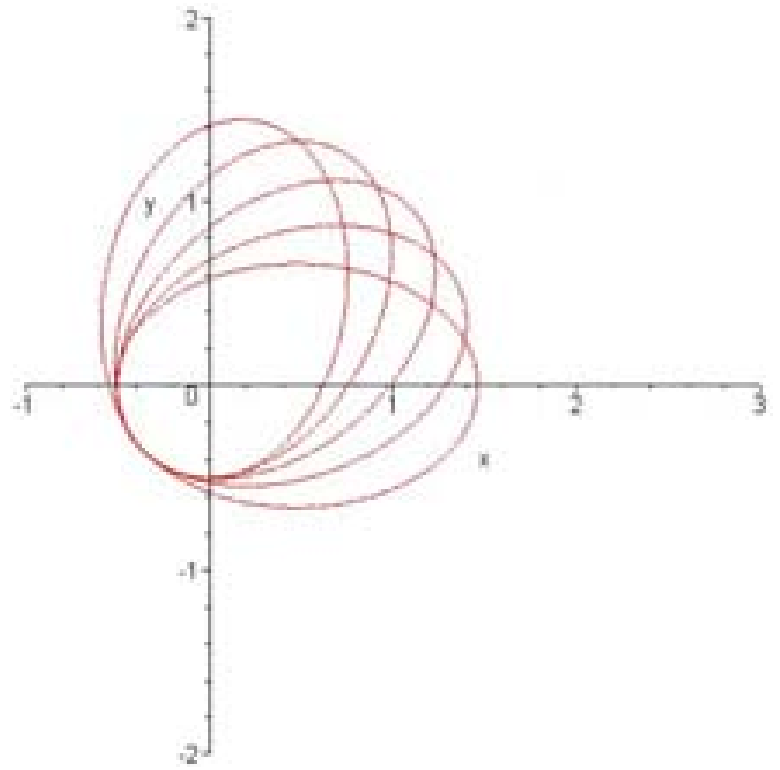

Figure 9c. Plot of an ellipse with progressive variation of the axes some angle $\rho$. 